Research Paper

\title{
In vitro study of new combinations for local antibiotic therapy with calcium sulphate - Near constant release of ceftriaxone offers new treatment options
}

\author{
Peter Wahl ${ }^{1,2}{ }^{\bowtie}$, Karolin Rönn ${ }^{1,3}$, Marc Bohner4, Laurent A Decosterd5, Christoph Meier², Michel Schläppi², \\ Sandrine Festa ${ }^{1}$, Emanuel Gautier ${ }^{1}$ \\ 1. Department of Orthopaedic Surgery, HFR Fribourg - Cantonal Hospital, Fribourg, Switzerland; \\ 2. Division of Orthopaedics and Traumatology, Cantonal Hospital Winterthur, Winterthur, Switzerland; \\ 3. Schulthess Clinic, Zürich, Switzerland; \\ 4. RMS Foundation, Bettlach, Switzerland; \\ 5. Division of Clinical Pharmacology, University Hospital Centre CHUV, Lausanne, Switzerland. \\ $\square$ Corresponding author: Dr Peter Wahl, Division of Orthopaedics and Traumatology, Cantonal Hospital Winterthur, Brauerstrasse 15, P.O. Box 834, 8401 \\ Winterthur, Switzerland. \\ (C) Ivyspring International Publisher. This is an open access article distributed under the terms of the Creative Commons Attribution (CC BY-NC) license \\ (https://creativecommons.org/licenses/by-nc/4.0/). See http://ivyspring.com/terms for full terms and conditions.
}

Received: 2018.03.21; Accepted: 2018.09.17; Published: 2018.10.04

\begin{abstract}
Introduction: Local application of antibiotics provides high concentrations at the site of interest, with minimal systemic toxicity. Carrier materials might help manage dead space. Calcium sulphate $\left(\mathrm{CaSO}_{4}\right)$ has a dissolution time that only slightly exceeds the usually recommended duration of systemic antibiotic treatments. This in vitro study evaluates compatibility, release kinetics and antibacterial activity of new combinations of antibiotics with $\mathrm{CaSO}_{4}$ as carrier material.

Methods: $\mathrm{CaSO}_{4}$ pellets added with $8 \% \mathrm{w} / \mathrm{w}$ antibiotic powder were exposed once in phosphate-buffered saline (PBS) solution and once in bovine plasma, in an elution experiment run over 6 weeks at $37^{\circ} \mathrm{C}$. Antibiotic elution was examined at various time points. Concentration was measured by liquid chromatography with tandem mass spectrometry. Antimicrobial activity was checked with an agar diffusion test.

Results: Piperacillin-tazobactam, ceftazidime, cefepime, and meropenem showed fast reduction of concentration and activity. Flucloxacillin and cefuroxime remained present in relevant concentrations for 4 weeks. Ciprofloxacin, levofloxacin and clindamycin lasted for 6 weeks, but also at cell toxic concentrations. Ceftriaxone showed a near-constant release with only a small reduction of concentration from 130 to $75 \mathrm{mg} / \mathrm{l}$. Elution profiles from PBS and plasma were comparable.

Conclusion: $\mathrm{CaSO}_{4}$ provides new possibilities in the local treatment of bone and joint infections. Ceftriaxone appears to be of particular interest in combination with $\mathrm{CaSO}_{4}$. Release persists at clinically promising concentrations, and appears to have a depot-like slow release from $\mathrm{CaSO}_{4}$, with only a small reduction in activity and concentration over 6 weeks. To the best of our knowledge, such a particular persistent release never was described before, for any antibiotic in combination with a carrier material for local application.
\end{abstract}

Key words: Calcium sulphate; $\mathrm{CaSO}_{4}$; in vitro elution; antibiotics; ceftriaxone

\section{Introduction}

In orthopaedic and trauma surgery, local application of antibiotics allows drug delivery in compartments poorly accessible to systemic administration, and the carrier material can help manage dead space [1-3]. Over the last decades, polymethylmethacrylate (PMMA) bone cement has 
become the standard of care carrier material $[1,2,4,5]$. However, PMMA is not resorbable, implying the disadvantages of incomplete drug release and requirement of secondary removal [2, 5-7]. For many practical and historical reasons, the antibiotics added to PMMA are mostly limited to glycopeptides, such as vancomycin and teicoplanin, aminoglycosides, such as gentamicin or tobramycin, or clindamycin $[1,4,5$, 8].

Longer lasting release kinetics than from PMMA would be desirable, as a very high initial peak is usually followed by prolonged and mostly subtherapeutic release after some days [9-12]. Calcium sulphate $\left(\mathrm{CaSO}_{4}\right)$ is a material widely used as a bone void filler and carrier for local delivery of antibiotics, with the particular advantage of resorbing within months $[2,13]$. Promising clinical experience with this carrier material has already been reported, both with aminoglycosides and vancomycin $[2,7,12$, 14, 15]. Furthermore, its compatibility with many more antibiotics has already been described as well [16-23]. However, most studies only examined short-term release. Studies testing release over 6 weeks, a duration of treatment usual for bone and joint infections, are not so common [22-25]. Regarding the complexity of the spectrum of bacteria encountered in bone and joint infections, a larger choice of antibiotics would be favourable [26-28].

This study aims at describing compatibility with the carrier material and release kinetics in the setting of an in vitro elution experiment of new combinations of antibiotics added to $\mathrm{CaSO}_{4}$. In contrast to most other studies, this experiment provides observations over a prolonged, clinically relevant period of 6 weeks. The effect of using a biological elution fluid, instead of simple electrolytic solutions, is tested as well, since the concomitant presence of proteins may significantly reduce the antibacterial effect of antibiotics [29].

\section{Methods}

\section{Preparation of the pellets}

$\mathrm{CaSO}_{4}$ hemihydrate was specifically produced for this experiment (lot E09-0019, RMS Foundation, Bettlach, Switzerland). For the sake of clarity, the powdered, dehydrated form will be denominated $\mathrm{CaSO}_{4}$ hemihydrate, while $\mathrm{CaSO}_{4}$ will be used for the dihydrate. $\mathrm{CaSO}_{4}$ in analytical quality (Ref. 12056, lot 70660, Honeywell Riedel-de-Haen, Seelze, Germany) was autoclaved four times at $134^{\circ} \mathrm{C}$ for $60 \mathrm{~min}$, with drying performed at $105{ }^{\circ} \mathrm{C}$. The powder was then grinded in a disk mill (Pulverisette 13, Fritsch, Idar-Oberstein, Germany), followed by milling for 5 min at 400 RPM in a planetary mill (Pulverisette 5,
Fritsch, Idar-Oberstein, Germany) adding 100\% w/w zirconia beads and $20 \% \mathrm{w} / \mathrm{w}$ ethanol. To confirm the hemihydrate nature, a sample of the powder was heated up at $400{ }^{\circ} \mathrm{C}$ (N20/14 furnace, Nabertherm, Germany). The resulting weight loss was $6.1 \%$, which is close to the theoretical value of $6.2 \%$ expected for the dehydration of $\mathrm{CaSO}_{4}$ hemihydrate into the anhydrous form. According to x-ray diffraction analysis (PW1800 x-ray diffractometer, PANalytical, Almelo, Netherlands), the powder contained 88\% hemihydrate, $5 \%$ dihydrate and $7 \%$ anhydrate. Electron-beam microscopy (S360, Leica Microsystems, Wetzlar, Germany), confirmed the typical rod-like appearance of alpha-hemihydrate.

Pellets were manufactured using silicone moulds, as described elsewhere $[12,30]$. The $\mathrm{CaSO}_{4}$ hemihydrate was mixed manually with the antibiotic powder, until macroscopically evenly blended. The antibiotics used are listed in Table 1. Note that drugs for medical use might contain excipients and preservatives [31]. The proportion of antibiotic to $\mathrm{CaSO}_{4}$ hemihydrate was $8 \%: 92 \% \mathrm{w} / \mathrm{w}$. Weighting was done on a microgram balance (XS203S, Mettler-Toledo, Greifensee, Switzerland). Mean error for antibiotic and $\mathrm{CaSO}_{4}$ hemihydrate weighting was $0.5 \%$ (SD 0.004) and 0.03\% (SD 0.0005), respectively. At least 35\% twice demineralized water was added to obtain a mouldable dough. Preparations with ceftriaxone required adding approximately twice as much water. Excess water was removed by drying the pellets in a dessicator for 36 hours. Despite a prolonged dessication process, imipenem-cilastatin hindered hardening of the $\mathrm{CaSO}_{4}$, and had to be discarded from further tests.

\section{Elution experiment}

For every antibiotic, $5 \mathrm{~g}$ of pellets were placed in each of four closed glass tubes. The elution experiment was run in triplicate, while the fourth tube allowed sampling of whole pellets for agar diffusion tests. Each tube was filled with $10 \mathrm{ml}$ of elution fluid, exchanged daily by pouring it out and refilling. As the aim of the experiment was to assess stability and activity of the antibiotics over a prolonged period of time, no thorough rinsing of the pellets was performed in order to avoid accidental losses of material and drug washout. The experiment was run once with isotonic phosphate-buffered saline (PBS, art. G227, lot 0922327, Grogg Chemie, Stettlen-Deisswil, Switzerland), and then repeated with bovine plasma (IBV-K2 EDTA, lot 11035, Innovative Research, Novi, MI, USA) for those antibiotics that showed prolonged stability in the first phase. Except for the required daily manipulations, the tubes where kept at $37^{\circ} \mathrm{C}$ for a total of 42 days. 
The same person (S.F.) performed all manipulations every day for the whole experiment, thus ensuring technical continuity. Enoxaparin-sodium (Clexane, Sanofi-Aventis, Meyrin, Switzerland) was added at $0.01 \mathrm{mg} / \mathrm{ml}$ to the plasma to avoid coagulation in the presence of $\mathrm{CaSO}_{4}$. Preliminary tests had been performed to verify dosing and to exclude interference with antibiotic assays.

\section{Antibiotic assays}

Concentrations of the antibiotics were measured by high performance liquid chromatography coupled with tandem mass spectrometry (LC-MS/MS) from samples collected daily for the first 8 days, on day 10 and 14, and then weekly until day 42 . One $\mathrm{ml}$ of elution fluid was sampled from every test tube in polypropylene cryotubes (Nunc, Thermo Scientific, Reinach, Switzerland), immediately shock-frozen in ethanol/dry ice, and then stored at $-80{ }^{\circ} \mathrm{C}$. After thawing, assaying was performed as a batch at the end of each phase of the experiment. All measures were done trice. Technical details have been published previously [8]. The lower limits of quantification (LOQ) of every antibiotic is listed in Table 1.

Antibacterial activity of the antibiotics was verified by Kirby-Bauer disc diffusion tests on days 1 , $2,3,5,7,10,14$ and then weekly until day 42. Dry paper discs $6 \mathrm{~mm}$ in diameter (Becton Dickinson, Heidelberg, Germany) were impregnated with $25 \mu 1$ of elution fluid and placed on Mueller Hinton agar (Biomérieux, Geneva, Switzerland) plates inoculated with an appropriate reference bacterium (Table 1), following the EUCAST protocol [8, 32, 33]. These assays were performed continuously during the experiment. A whole pellet was also sampled from the fourth tube, and placed directly on the agar. Inhibition zones were measured at the nearest $\mathrm{mm}$ after incubation for one day at $37^{\circ} \mathrm{C}$.

\section{Statistical analysis}

Statistical analyses were performed using SPSS Version 21 (SPSS Inc., Chicago, IL, USA). All results are presented as means of triplicate values and standard deviation (SD). A paired t-test was employed to assess the differences in antibiotic concentration between PBS and plasma. The level of significance was set at $p<0.05$. Graphical illustrations were made with Illustrator CS6 Version 16 (Adobe Systems, San Jose, California, USA). Simulations of release kinetics were performed with Excel 2010 (Microsoft, Redmond, Washington, USA), considering solubility of the antibiotics [34] and time to equilibrium.

\section{Results}

Results from determination of concentration by LC-MS/MS of piperacillin, tazobactam, ceftazidime, cefepime and meropenem are illustrated in Figure 1. Corresponding results from disc diffusion tests of these antibiotics are presented in Figure 2. As these antibiotics showed a relatively rapid elimination, they were not considered for further testing in plasma.

Results for flucloxacillin, cefuroxime, ceftriaxone, ciprofloxacin, levofloxacin and clindamycin, including statistical analysis, are presented in Figure 3 for quantification by LC-MS/MS and Figure 4 for inhibition zone size from disc diffusion tests. P-values $<0.05$ could be identified for flucloxacillin, cefuroxime, ciprofloxacin and clindamycin. A major and potentially relevant difference could however only be observed for ciprofloxacin. Except for ciprofloxacin, results from the plasma test series tended towards higher concentrations than in PBS. Regarding the disc diffusion tests as shown in Figure 4, direct comparison from one antibiotic to another shall not be made as different reference organisms have been used, depending on the antibiotic spectrum of each drug.

Table 1. List of antibiotics used in the experiments. The lower limit of quantification (LOQ) of the LC-MS/MS assay used is listed. The reference bacterium used for disc diffusion assays is also included.

\begin{tabular}{|c|c|c|c|c|}
\hline Antibiotic & Product Table & Supplier & LC-MS/MS LOQ & Disk Diffusion \\
\hline Flucloxacillin & Floxapen $^{\circledR}$ & Actavis, Regensdorf, Switzerland & $0.1 \mathrm{mg} / \mathrm{L}$ & S. aureus ATCC 25923 \\
\hline Piperacillin+tazobactam & Tazobac $^{\circledR}$ & Actavis, Regensdorf, Switzerland & $\begin{array}{l}\text { Piperacillin } 0.08 \mathrm{mg} / \mathrm{L} \\
\text { Tazobactam } 0.05 \mathrm{mg} / \mathrm{L}\end{array}$ & E.coli ATCC 35218 \\
\hline Cefuroxime & Zinacef ${ }^{\circledR}$ & GlaxoSmithKline, Münchenbuchsee, Switzerland & $0.1 \mathrm{mg} / \mathrm{L}$ & E. coli ATCC 25922 \\
\hline Ceftriaxone & Rocephin $^{\circledR}$ & Roche Pharma, Reinach, Switzerland & $0.5 \mathrm{mg} / \mathrm{L}$ & E. coli ATCC 25922 \\
\hline Ceftazidime & Fortam $^{\circledR}$ & GlaxoSmithKline, Münchenbuchsee, Switzerland & $0.1 \mathrm{mg} / \mathrm{L}$ & E. coli ATCC 25922 \\
\hline Cefepime & Cefepime OrPha ${ }^{\circledR}$ & OrPha Pharma, Küsnacht, Switzerland & $0.05 \mathrm{mg} / \mathrm{L}$ & E. coli ATCC 25922 \\
\hline Imipenem+cilastatin & Tienam $^{\circledR}$ & MerckSharp\&Dohme, Lucerne, Switzerland & Imipenem $0.05 \mathrm{mg} / \mathrm{L}$ & E. coli ATCC 25922 \\
\hline Meropenem & Analytical quality & AstraZeneca, Grafenau, Switzerland & $0.05 \mathrm{mg} / \mathrm{L}$ & E. coli ATCC 25922 \\
\hline Ciprofloxacin & Analytical quality & Bayer, Zürich, Switzerland & $0.1 \mathrm{mg} / \mathrm{L}$ & E. coli ATCC 25922 \\
\hline Levofloxacin & Analytical quality & Sanofi-Aventis, Vernier, Switzerland & $0.1 \mathrm{mg} / \mathrm{L}$ & E. coli ATCC 25922 \\
\hline Clindamycin & Analytical quality & Pfizer, Zürich, Switzerland & $0.1 \mathrm{mg} / \mathrm{L}$ & S. aureus ATCC 25923 \\
\hline
\end{tabular}




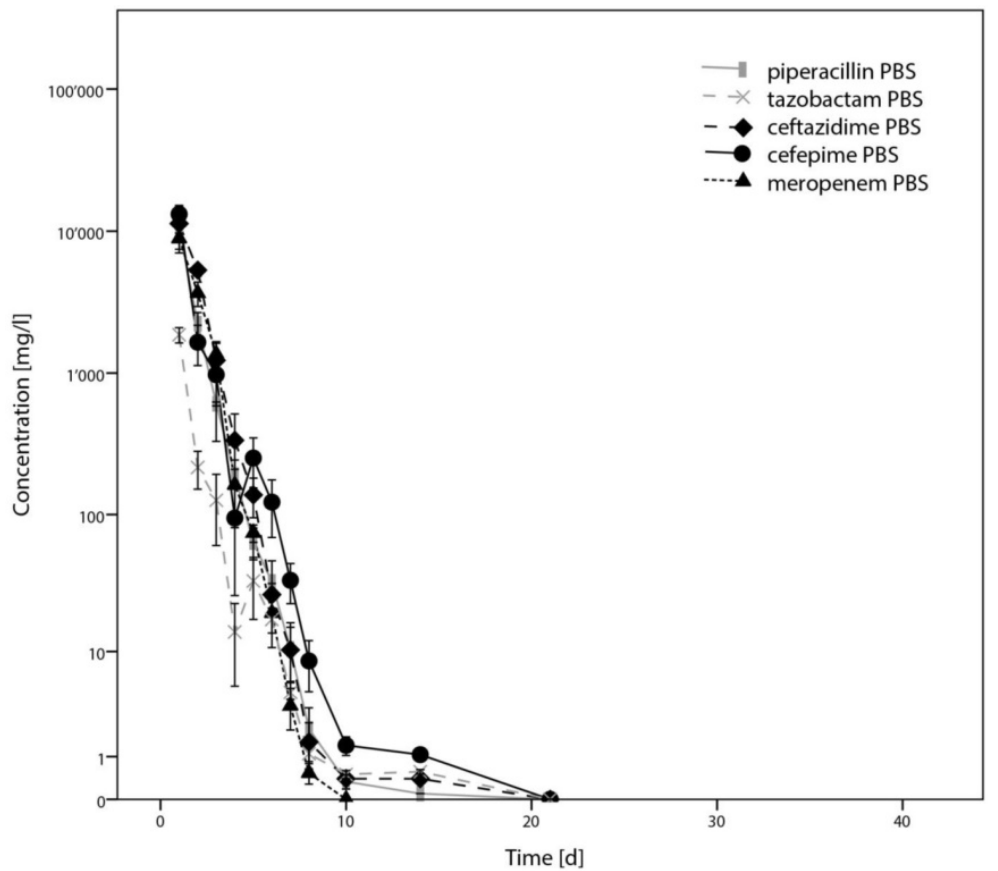

Figure 1. Results from determination of concentration of piperacillin/tazobactam, ceftazidime, cefepime and meropenem by HPLC-MS, presented as means from triplicates, with error bars indicating standard deviations.

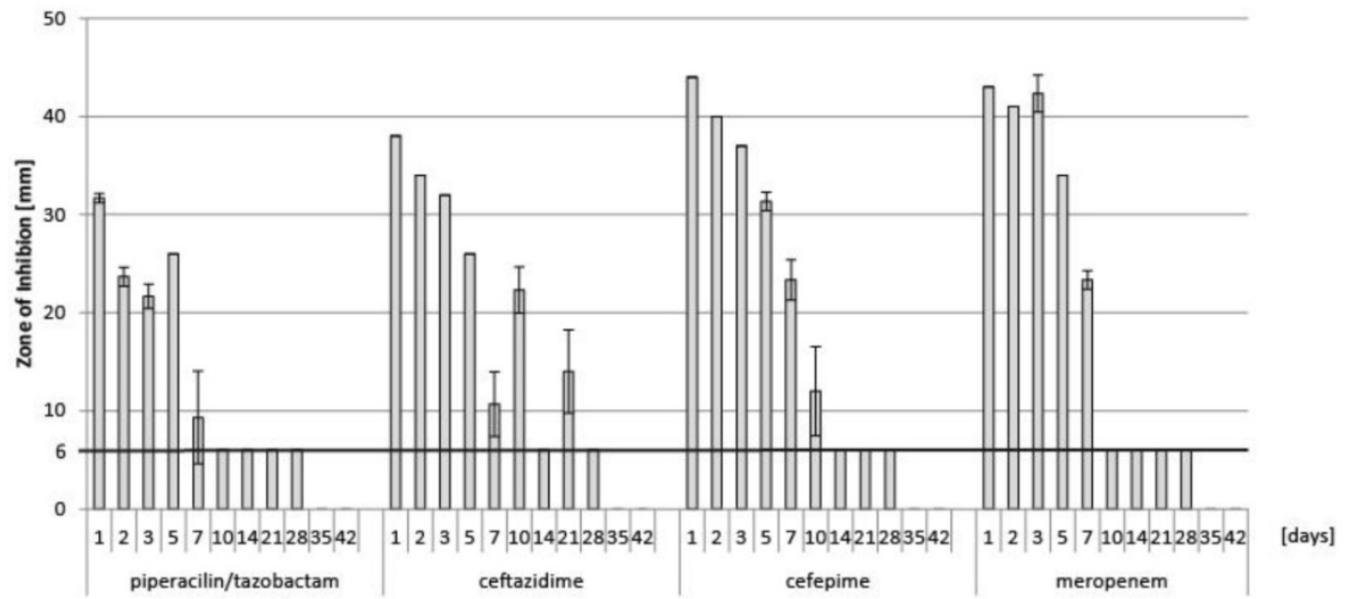

Figure 2. Results from determination of antibacterial activity of piperacillin-tazobactam, ceftazidime, cefepime and meropenem by disc diffusion test from the test series in PBS. Piperacillin and tazobactam had to be tested in combination for this assay. Data are presented as means of triplicates of the diameter of the zone of inhibition in mm, with error bars indicating standard deviation. The horizontal line at $6 \mathrm{~mm}$ indicates the diameter of the paper disc.

Results from disc diffusion assays with an entire pellet are not presented, as they provided congruent results with diffusion from paper discs, with technical issues limiting interpretability.

Sample results from the release simulations are illustrated in Figure 5, along with correlations of observed results from the elution experiment.

\section{Discussion}

This study provides some new insights useful in optimizing local application of antibiotics to treat bone, joint, and implant-associated infections. In contrast to all the other tested antibiotic agents, ceftriaxone showed an elution profile corresponding to a slow release when used with $\mathrm{CaSO}_{4}$ as carrier material.

For application as a bone void filler or antibiotic carrier, $\mathrm{CaSO}_{4}$ hemihydrate in its alpha variant is generally preferred over the beta variant, as it provides a dehydrate less porous with a more predictable resorption behaviour $[13,35,36]$. While a product not commercially available was used for this experiment, internal verification confirmed that it was an alpha-hemihydrate of $\mathrm{CaSO}_{4}$. Determination of the exact proportion of the tested antibiotics in the final pellets would require a complex chemical analysis, as water has to be added in excess during preparation of $\mathrm{CaSO}_{4}$, which evaporates during the curing process. However, the technique described in this study produces pellets containing approximately as much 
antibiotic as commonly used in clinical application [2, $7,12,14,15,30]$. The very high porosity of $\mathrm{CaSO}_{4}(>2$ $\mathrm{m}^{2} / \mathrm{g}$ ) [36] might have been increased due to the addition of antibiotics, as it has been demonstrated for other materials $[6,37]$. Increasing the concentration of antibiotics increases release from $\mathrm{CaSO}_{4}$, probably due to increased porosity $[19,22]$. This is well known for PMMA, and increasing porosity in such a way, and others, is used to increase antibiotic release in
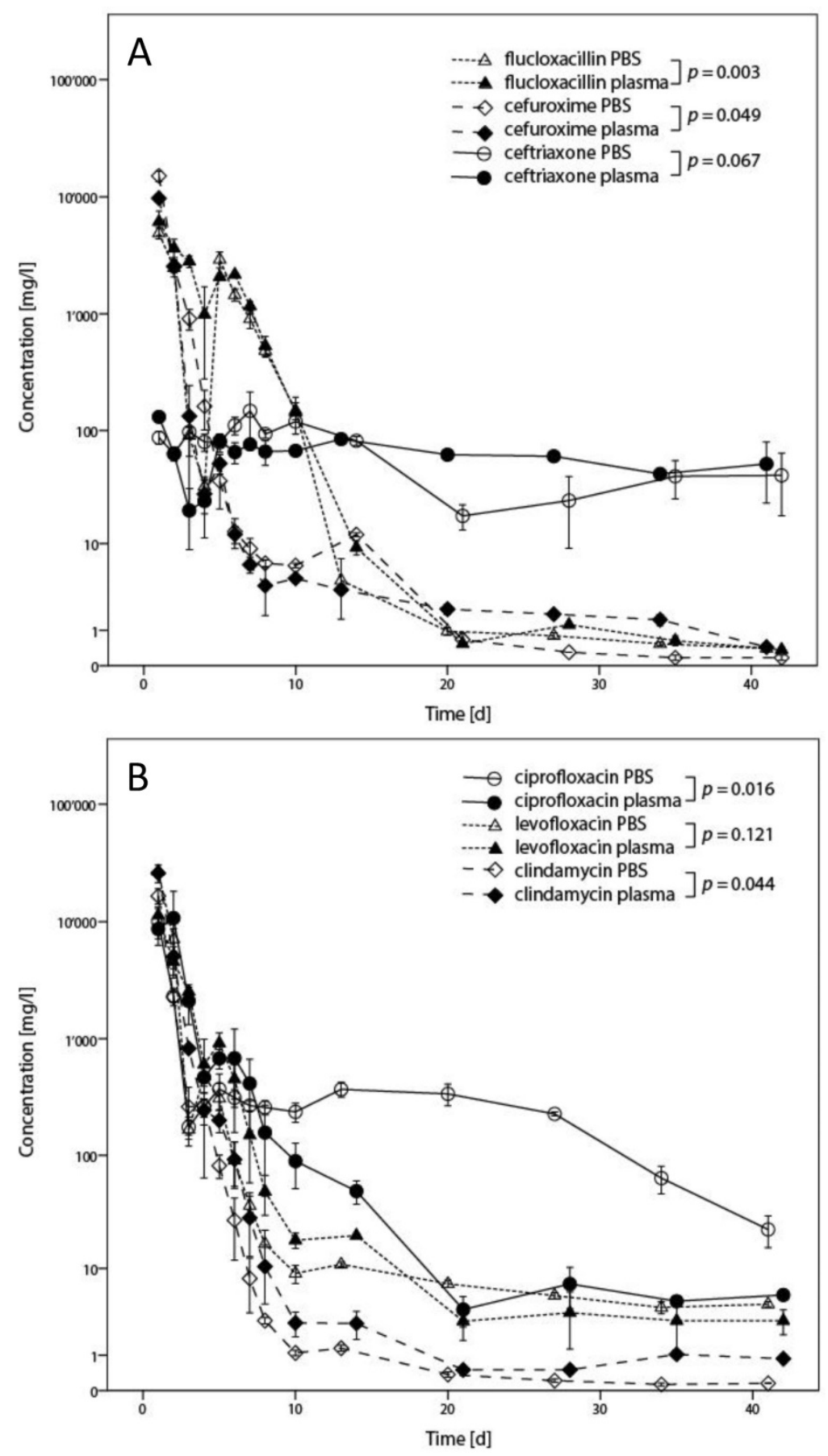

Figure 3. Results from determination of concentration in the elution fluids by HPLC-MS indicated for both PBS and plasma. In Fig. 3A, results for flucloxacillin, cefuroxime, and ceftriaxone are presented. Fig. $3 \mathrm{~B}$ shows the results for ciprofloxacin, levofloxacin and clindamycin. Results are presented as means from triplicates with error bars indicating standard deviation, with statistical analysis of the comparison between results in PBS and plasma indicated in the legend. clinical application [6, 38-40]. Even if the ratio of external surface to volume influences drug elution from $\mathrm{CaSO}_{4}$, porosity remains the main material-based predictor of antibiotic elution in calcium-based carriers [22, 23, 41]. With the exception of a potential calcium salt formation, there is no covalent binding of the added drug. Thus, a large proportion of the antibiotic is usually free to diffuse. Technical variability, such as inhomogeneity of the pellets and residual fluid during daily exchange, most likely explains differences in concentrations observed between PBS and plasma for flucloxacillin, cefuroxime and clindamycin (Figures $3 \& 4$ ), even if statistically significant differences could be identified for these antibiotics. Technical issues probably had an influence on results more important than many molecular aspects.

Elution experiments test many factors simultaneously. First, the compatibility of the drug with the chosen carrier material, particularly degradation of the drug due to any heat production during the manufacturing process $[8,42]$. Second, the stability of the drug as it is exposed to the chosen physicochemical conditions over time. Most antibiotics suffer significant degradation being exposed to body temperature in aqueous solution $[8,21,43$, 44]. Third, exchange of the elution fluid greatly influences elimination of the drug, particularly for highly soluble molecules $[42,45]$. In the setting of the current study, approximately $90 \%$ of the fluid has been exchanged on a daily basis, the rest remaining trapped among the pellets. All drugs tested, except ceftriaxone, showed an initial high peak, with a more or less rapid decrease of concentration and activity over time (Figures 1 \& 3). Considering subtotal daily fluid exchange, rapid decrease of concentration and activity can be explained by either rapid release or rapid degradation of the drug at body temperature in aqueous solution $[8,43,44]$. Rapid release depends on high solubility [42], and the fact that the carrier material does not bind the drug, which would delay its release [12]. The influence of solubility on antibiotic concentrations in an elution experiment is illustrated in Figure 5, where simulated release curves are compared to observed results. While most profiles correspond to agents with intermediate 
solubility, ceftriaxone shows an elution profile corresponding to a slow release limited by its solubility. Although antibiotic concentrations well above common minimal inhibitory concentrations have been observed for several weeks for flucloxacillin, cefuroxime, ciprofloxacin, levofloxacin and clindamycin in our experiment, their clinical significance remains unclear. The kinetics of wound fluid are unknown and may by different to this experimental setting. It can also be expected that any carrier material will be incorporated in vivo in a blood clot, which might alter elution kinetics, even if an equilibrium within the local compartment might be expected due to the slow release [46]. Generalisation beyond the general pharmacokinetic behaviour of the observed results into clinical practice remains limited.
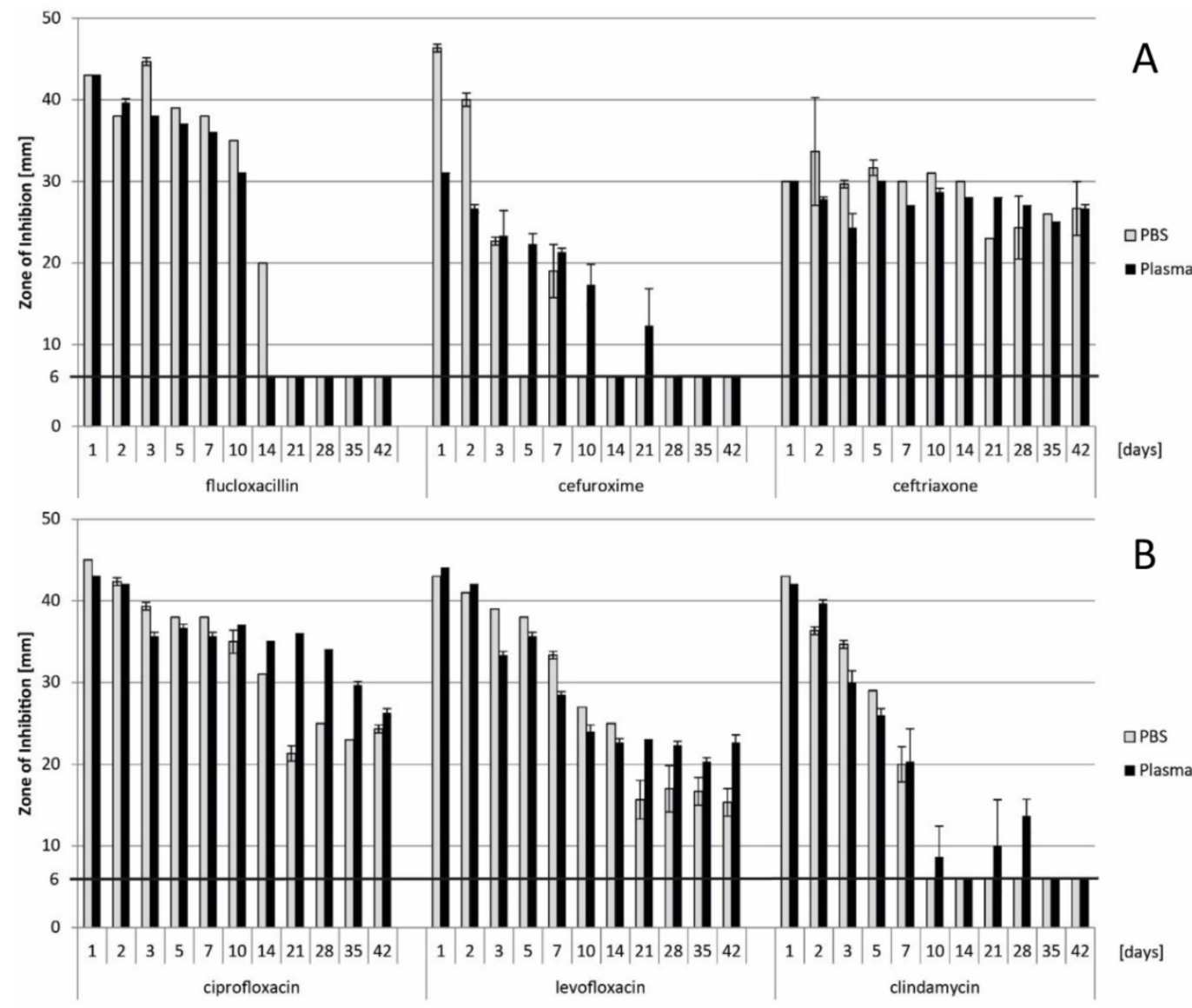

Figure 4. Results from determination of antibacterial activity by disc diffusion test, indicating the diameter of the zone of inhibition in mm. Data presented as means from triplicates, with error bars indicating standard deviation. In Fig. 4A, results of flucloxacillin, cefuroxime and ceftriaxone are shown. Fig. 4B provides the results for ciprofloxacin, levofloxacin and clindamycin. The horizontal line at $6 \mathrm{~mm}$ indicates the diameter of the paper disc. Direct comparison from one antibiotic to another shall not be made as different reference organisms have been used.

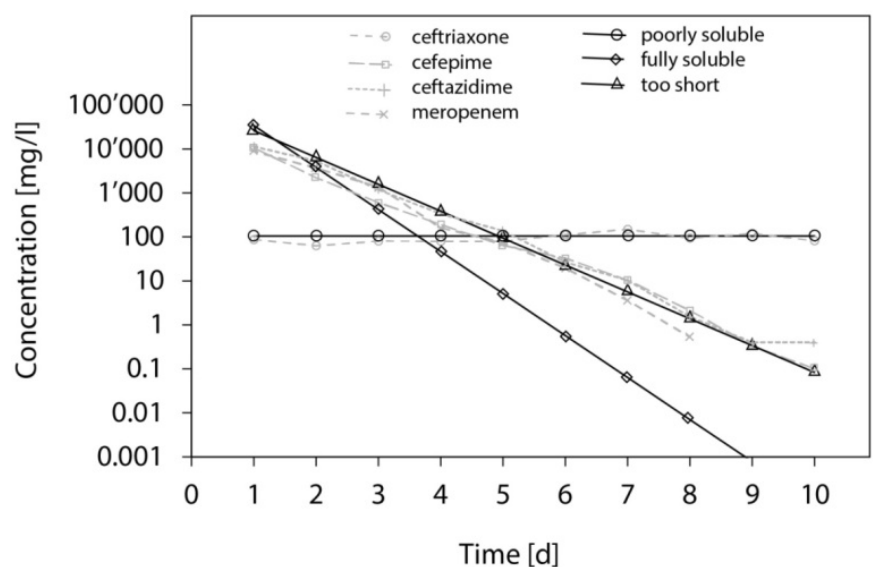

Figure 5. Calculation of the expected antibiotic concentrations after each incubation step using three assumptions: (o) the solubility of the drug is limited to $105 \mathrm{mg} / \mathrm{L}$ (e.g. Ceftriaxone); $(\Delta)$ the drug is fully soluble and the system is fully equilibrated at the end of each incubation; ( $\square$ ) the drug is fully soluble, but only $85 \%$ of the drug have time to diffuse out of the pellets at the end of each incubation. To assess the validity of these calculations, some of the experimental results are plotted: $(0)$ Ceftriaxone; $(\mathbf{x})$ Cefepime; $(\boldsymbol{\Delta})$ ceftazidime; $(+)$ meropenem. 
In contrast to the other tested antibiotic agents, ceftriaxone shows a near-constant release. Despite additional water added during preparation, which is expected to have increased porosity [41]. This slow release can only be explained by formation of a depot within the $\mathrm{CaSO}_{4}$. When exposed in aqueous solution without $\mathrm{CaSO}_{4}$, this drug shows an intermediate stability, with near-complete degradation during the observation period of this study [8,44]. Ceftriaxone, usually provided as a disodium salt, is known to bind and precipitate with $\mathrm{Ca}^{++}$ions. This mechanism is implicated in the formation of biliary sludge or urolithiasis during treatment with ceftriaxone and caused complications due to in vivo precipitation of the drug after concomitant administration of both substances [47-49]. The kinetic solubility constant of ceftriaxone and calcium at $37^{\circ} \mathrm{C}$ has been described as being between $0.162^{*} 10^{-5} \mathrm{~mol} / \mathrm{l}^{2}$ in crystalloid solution [49] and $4.2^{*} 10^{-5} \mathrm{~mol} / \mathrm{l}^{2}$ in plasma [47], with differences accounted for by considerations of the matrix and the proportion of protein-binding in biological fluids. This corresponds to a maximum solubility of ceftriaxone between 705 and 3'594 mg/l, considering a molecular weight of $554.571 \mathrm{~g} / \mathrm{mol}$ for ceftriaxone. However, precipitation of ceftriaxone in the presence of calcium is a complex phenomenon, as this couple has a wide metastable range, exceeding the solubility product by a factor of 10, favourably influenced by biological fluids [47-49]. Saturation of the solution is certainly exceeded when $\mathrm{CaSO}_{4}$ pellets are manufactured. The concentrations observed in the current experiment do not show a decrease as it would be if induced by hydrolysis at body temperature in aqueous solution $[8,44]$. In addition, the observed concentrations remain around $100 \mathrm{mg} / \mathrm{l}$ after $24 \mathrm{~h}$ of elution, which is far below the concentrations required to observe precipitation in biological fluids [47-49]. Thus, slow release from a depot of Ca-ceftriaxone can be postulated, with solubility limited by $\mathrm{Ca}^{++}$-saturation of the solution. To the best of our knowledge, this near-constant release over a long period has not been described so far, neither for ceftriaxone, nor for any other antibiotic carrier material used for local antibiotic application in orthopaedic surgery. The observed slow release of ceftriaxone from $\mathrm{CaSO}_{4}$ pellets would provide optimal kinetics, with sufficient drug concentration for a prolonged time of exposure. This is the essential pharmacodynamic parameter determining bacterial killing and treatment success by beta-lactam antibiotics in general, and ceftriaxone in particular $[29,50]$.

Particular strengths of this study are the length of the observation period, the use of a biological fluid, and that two different analytical methods have been used. It remains unknown how long exactly local antibiotics are required. In the current experiment, a period of 6 weeks had been chosen as it corresponds to commonly recommended duration of treatment protocols for bone and implant-associated infections $[24,25]$. in vitro evidence supports that similar durations are required to eradicate biofilm by the action of antibiotics alone [11]. The experiments have been repeated in plasma in order to illustrate potential differences on antibiotic disposition between crystalloid and complex biological fluids. Protein binding of antibiotics has a known relevant effect on bacterial killing $[29,51]$. However, only ciprofloxacin showed a marked decrease of concentration in plasma (Figure 3B). This was apparent only in LC-MS/MS assays. The decreased concentration might be due either to protein binding or to binding with calcium ions [51]. Nevertheless, no decrease of the antibacterial activity could be observed, but this might also be explained by a lack of sensitivity of disc diffusion tests (Figure 4). For the other antibiotics, the observed differences were smaller than the variability of the methods, even if statistical tests showed significant results. This is illustrated by the fact that the curves cross each other and that differences mostly are smaller than a $95 \%$ confidence interval of approximately 2 standard deviations would be. Differences between results from PBS and plasma also appear less than what might be expected as clinically relevant, whereas both test series show similar behaviours.

While antibiotic concentration has been determined by LC-MS/MS, antibacterial activity has been verified by disc diffusion test. Even if LC-MS/MS is a very specific method that should detect alterations of the molecule [52], biological activity of the tested drugs has been investigated as well. Both methods provided congruent results. However, results from the disc diffusion assays could not be used for quantification. The establishment of a calibration curve and serial dilution would have been required to ensure that measurements are performed in the window of linear dose-response [8, 53]. However, this additional analysis would have exceeded the setting and feasibility of this study. Interpretation of results from disk diffusion tests using entire pellets remains limited by many technical issues. Firstly, the quantity of antibiotics contained in the pellets was not standardized and decreased over time with dissolution of the carrier and elution of the drug. Secondly, contact of the pellets with the surface of the agar showed major variability. And lastly, a relevant number of pellets did not adhere sufficiently to the agar without breaking it and interrupting 
diffusion of the drug. For these reasons, results have not been shown in detail.

Plasma had been chosen instead of serum, as coagulation factors make up a large proportion of the protein content of blood. However, dissolution of $\mathrm{CaSO}_{4}$ saturates the solution with $\mathrm{Ca}^{++}$ions, which are essential catalysts in various steps of the coagulation cascade [54]. Low molecular weight heparins were added to the plasma to avoid coagulation. While heparins with lithium ions are known to cause a matrix effect that might influence results from LC-MS/MS [55], the heparin used was enoxaparin-Na. Nevertheless, the absence of influence on results was verified for ceftriaxone, even if results from this test series are not shown. LC-MS/MS has a higher specificity than fluorescence polarisation assays, which are still standard in clinical routine for therapeutic drug monitoring $[52,56]$. The results from disc diffusion assays are not influenced, considering comparable results between PBS and plasma series. But this might be solely due to the low sensitivity of this method.

Most quantification results showed a shouldering at 5-7 days. Technical issues probably explain this phenomenon. Even if all manipulations regarding the elution part of the experiment have been performed by the same person, variability of residual fluid in the test tubes at daily fluid exchange is the most probable explanation. Progressive alteration of the consistency of the pellets may have caused some difficulties during vortexing and fluid exchange, which could not be quantified in this study. As the pellets were manufactured manually, the material certainly suffered from some degree of inhomogeneity. This fact would certainly provide a very probable explanation for this shouldering as it was simultaneously observed for all tested antibiotic agents. Storage should not be an issue as the samples were shock-frozen and kept at $-80^{\circ} \mathrm{C}$ until assaying, a temperature that ensures stability of even the most fragile beta-lactams [43].

Last but not least, when discussing the choice of antibiotics for local application, cellular toxicity thresholds must be considered. This is particularly problematic for the quinolones [57-59]. Vancomycin, clindamycin, the aminoglycosides, and to a slightly lesser extend all beta-lactam antibiotics, all show low toxicity, offering broad margins for local application [58-60]. Although there is no such specific data available in the literature for ceftriaxone, the concentrations observed remained far below the toxicity threshold known for other cephalosporins [59]. Cell toxicity however is a time-dependant phenomenon $[57,58,60]$. Only suppositions can be made regarding toxicity of a prolonged exposure over weeks, as this never has been published to the best of our knowledge.

In conclusion, this study not only provides new insights allowing optimization of local antibiotic application with $\mathrm{CaSO}_{4}$ as a carrier material, but it also illustrates limits of classic elution experiments. Ceftriaxone appears to have a particular behaviour, not described so far, with a near-constant release at $\sim 100 \mathrm{mg} / 1$ for the 6 weeks duration of the experiment. This observation may open new possibilities for the treatment of bone and joint infections.

\section{Acknowledgements}

Funding for materials and assays was provided by the RMS Foundation, Bettlach, Switzerland. Complementary funding was provided by Pfizer, Zurich, Switzerland, and by Roche Pharma, Rheinach, Switzerland. Antibiotics used for the experiment were provided gratefully by the manufacturers, as mentioned.

\section{Author contribution}

Peter Wahl Conceptualization, data curation, formal analysis, funding acquisition, investigation, project administration, validation, visualization, writing of manuscript

Karolin Rönn Investigation, methodology, validation, review\&editing

Marc Bohner Conceptualization, formal analysis, funding acquisition, methodology, resources, review\&editing

Laurent A Decosterd Conceptualization, data curation, formal analysis, investigation, methodology, resources, validation, review\&editing

Christoph Meier Supervision, validation, review\&editing

Michel Schläppi Formal analysis, software, visualization, review\&editing

Sandrine Festa Investigation, methodology, resources, review\&editing

Emanuel Gautier Conceptualization, funding acquisition, project administration, resources, supervision, review\&editing

\section{Competing Interests}

The authors have declared that no competing interest exists.

\section{References}

1. Henry SL, Galloway KP. Local antibacterial therapy for the management of orthopaedic infections. Pharmacokinetic considerations. Clinical pharmacokinetics. 1995; 29: 36-45.

2. McKee MD, Li-Bland EA, Wild LM, Schemitsch EH. A prospective, randomized clinical trial comparing an antibiotic-impregnated bioabsorbable bone substitute with standard antibiotic-impregnated cement beads in the treatment of chronic osteomyelitis and infected nonunion. Journal of orthopaedic trauma. 2010; 24: 483-90. 
3. Landersdorfer CB, Bulitta JB, Kinzig M, Holzgrabe U, Sorgel F. Penetration of antibacterials into bone: pharmacokinetic, pharmacodynamic and bioanalytical considerations. Clinical pharmacokinetics. 2009; 48: 89-124.

4. Iarikov D, Demian H, Rubin D, Alexander J, Nambiar S. Choice and doses of antibacterial agents for cement spacers in treatment of prosthetic joint infections: review of published studies. Clinical infectious diseases : an official publication of the Infectious Diseases Society of America. 2012; 55: 1474-80.

5. Kanellakopoulou K, Giamarellos-Bourboulis EJ. Carrier systems for the local delivery of antibiotics in bone infections. Drugs. 2000; 59: 1223-32.

6. Hsieh PH, Tai CL, Lee PC, Chang YH. Liquid gentamicin and vancomycin in bone cement: a potentially more cost-effective regimen. The Journal of arthroplasty. 2009; 24: 125-30.

7. Chang W, Colangeli M, Colangeli S, Di Bella C, Gozzi E, Donati D. Adult osteomyelitis: debridement versus debridement plus Osteoset $\mathrm{T}$ pellets. Acta orthopaedica Belgica. 2007; 73: 238-43.

8. Samara E, Moriarty TF, Decosterd LA, Richards RG, Gautier E, Wahl P. Antibiotic stability over six weeks in aqueous solution at body temperature with and without heat treatment that mimics the curing of bone cement. Bone Joint Res. 2017; 6: 296-306.

9. Anagnostakos K, Wilmes P, Schmitt E, Kelm J. Elution of gentamicin and vancomycin from polymethylmethacrylate beads and hip spacers in vivo. Acta orthopaedica. 2009; 80: 193-7.

10. Hsieh PH, Huang KC, Tai CL. Liquid gentamicin in bone cement spacers: in vivo antibiotic release and systemic safety in two-stage revision of infected hip arthroplasty. The Journal of trauma. 2009; 66: 804-8.

11. Post V, Wahl P, Richards RG, Moriarty TF. Vancomycin displays time dependent eradication of mature Staphylococcus aureus biofilms. Journal of orthopaedic research : official publication of the Orthopaedic Research Society. 2016.

12. Wahl P, Guidi M, Benninger E, Ronn K, Gautier E, Buclin T, et al. The levels of vancomycin in the blood and the wound after the local treatment of bone and soft-tissue infection with antibiotic-loaded calcium sulphate as carrier material. Bone Joint J. 2017; 99-B: 1537-44.

13. Kelly CM, Wilkins RM, Gitelis S, Hartjen C, Watson JT, Kim PT. The use of a surgical grade calcium sulfate as a bone graft substitute: results of a multicenter trial. Clinical orthopaedics and related research. 2001: 42-50.

14. Ferguson JY, Dudareva M, Riley ND, Stubbs D, Atkins BL, McNally MA. The use of a biodegradable antibiotic-loaded calcium sulphate carrier containing tobramycin for the treatment of chronic osteomyelitis: a series of 195 cases. Bone Joint J. 2014; 96-B: 829-36.

15. Helgeson MD, Potter BK, Tucker CJ, Frisch HM, Shawen SB. Antibiotic-impregnated calcium sulfate use in combat-related open fractures. Orthopedics. 2009; 32: 323.

16. Wang Y, Wang X, Li H, Xue D, Shi Z, Qi Y, et al. Assessing the character of the rhBMP-2- and vancomycin-loaded calcium sulphate composites in vitro and in vivo. Archives of orthopaedic and trauma surgery. 2011; 131: 991-1001.

17. Richelsoph KC, Webb ND, Haggard WO. Elution behavior of daptomycin-loaded calcium sulfate pellets: a preliminary study. Clinical orthopaedics and related research. 2007; 461: 68-73.

18. Panagopoulos P, Tsaganos T, Plachouras D, Carrer DP, Papadopoulos A, Giamarellou $\mathrm{H}$, et al. in vitro elution of moxifloxacin and fusidic acid by a synthetic crystallic semihydrate form of calcium sulphate (Stimulan). International journal of antimicrobial agents. 2008; 32: 485-7.

19. Wichelhaus TA, Dingeldein E, Rauschmann M, Kluge S, Dieterich R, Schafer $\mathrm{V}$, et al. Elution characteristics of vancomycin, teicoplanin, gentamicin and clindamycin from calcium sulphate beads. The Journal of antimicrobial chemotherapy. 2001; 48: 117-9.

20. Bowyer GW, Cumberland N. Antibiotic release from impregnated pellets and beads. The Journal of trauma. 1994; 36: 331-5.

21. Mousset B, Benoit MA, Delloye C, Bouillet R, Gillard J. Biodegradable implants for potential use in bone infection. An in vitro study of antibiotic-loaded calcium sulphate. International orthopaedics. 1995; 19: $157-61$.

22. Roberts R, McConoughey SJ, Calhoun JH. Size and composition of synthetic calcium sulfate beads influence dissolution and elution rates in vitro. Journal of biomedical materials research Part B, Applied biomaterials. 2014; 102: 667-73.

23. Aiken SS, Cooper JJ, Florance H, Robinson MT, Michell S. Local release of antibiotics for surgical site infection management using high-purity calcium sulfate: an in vitro elution study. Surg Infect (Larchmt). 2015; 16: 54-61.

24. Stengel D, Bauwens K, Sehouli J, Ekkernkamp A, Porzsolt F. Systematic review and meta-analysis of antibiotic therapy for bone and joint infections. The Lancet Infectious diseases. 2001; 1: 175-88.

25. Osmon DR, Berbari EF, Berendt AR, Lew D, Zimmerli W, Steckelberg JM, et al. Diagnosis and management of prosthetic joint infection: clinical practice guidelines by the Infectious Diseases Society of America. Clinical infectious diseases : an official publication of the Infectious Diseases Society of America. 2013; 56: e1-e25

26. Schafer P, Fink B, Sandow D, Margull A, Berger I, Frommelt L. Prolonged bacterial culture to identify late periprosthetic joint infection: a promising strategy. Clinical infectious diseases : an official publication of the Infectious Diseases Society of America. 2008; 47: 1403-9.

27. Schwotzer N, Wahl P, Fracheboud D, Gautier E, Chuard C. Optimal culture incubation time in orthopedic device-associated infections: a retrospective analysis of prolonged 14-day incubation. Journal of clinical microbiology. 2014; 52: 61-6
28. Zuluaga AF, Galvis W, Saldarriaga JG, Agudelo M, Salazar BE, Vesga O. Etiologic diagnosis of chronic osteomyelitis: a prospective study. Archives of internal medicine. 2006; 166: 95-100.

29. Palmer SM, Kang SL, Cappelletty DM, Rybak MJ. Bactericidal killing activities of cefepime, ceftazidime, cefotaxime, and ceftriaxone against Staphylococcus aureus and beta-lactamase-producing strains of Enterobacter aerogenes and Klebsiella pneumoniae in an in vitro infection model. Antimicrob Agents Chemother. 1995; 39: 1764-71.

30. Gitelis S, Brebach GT. The treatment of chronic osteomyelitis with a biodegradable antibiotic-impregnated implant. Journal of orthopaedic surgery. 2002; 10: 53-60.

31. [Internet] HCI Solutions AG: compendium.ch by HCI Solutions. Web Version 1.0.0.15034. http://www.compendium.ch/

32. Matuschek E, Brown DF, Kahlmeter G. Development of the EUCAST disk diffusion antimicrobial susceptibility testing method and its implementation in routine microbiology laboratories. Clinical microbiology and infection : the official publication of the European Society of Clinical Microbiology and Infectious Diseases. 2014; 20: O255-66.

33. [Internet] EUCAST: Breakpoint tables for interpretation of MICs and zone diameters. Version 5.0. http://www.eucast.org/clinical_breakpoints/

34. [Internet] DrugBank: Breakpoint tables for interpretation of MICs and zone diameters. Version 5.1.1. http://www.drugbank.ca/

35. Thomas MV, Puleo DA, Al-Sabbagh M. Calcium sulfate: a review. Journal of long-term effects of medical implants. 2005; 15: 599-607.

36. Rauschmann MA, Wichelhaus TA, Stirnal V, Dingeldein E, Zichner L, Schnettler R, et al. Nanocrystalline hydroxyapatite and calcium sulphate as biodegradable composite carrier material for local delivery of antibiotics in bone infections. Biomaterials. 2005; 26: 2677-84

37. Kweon C, McLaren AC, Leon C, McLemore R. Amphotericin B delivery from bone cement increases with porosity but strength decreases. Clinical orthopaedics and related research. 2011; 469: 3002-7.

38. Hanssen AD, Spangehl MJ. Practical applications of antibiotic-loaded bone cement for treatment of infected joint replacements. Clinical orthopaedics and related research. 2004: 79-85.

39. Anguita-Alonso P, Rouse MS, Piper KE, Jacofsky DJ, Osmon DR, Patel R. Comparative study of antimicrobial release kinetics from polymethylmethacrylate. Clinical orthopaedics and related research. 2006; 445: $239-44$.

40. Samuel S, Mathew BS, Veeraraghavan B, Fleming DH, Chittaranjan SB, Prakash JA. in vitro study of elution kinetics and bio-activity of meropenem-loaded acrylic bone cement. Journal of Orthopaedics and Traumatology. 2012; 13: 131-6.

41. Bohner M, Lemaitre J, Van Landuyt P, Zambelli PY, Merkle HP, Gander B. Gentamicin-loaded hydraulic calcium phosphate bone cement as antibiotic delivery system. J Pharm Sci. 1997; 86: 565-72

42. Zilberman M, Elsner JJ. Antibiotic-eluting medical devices for various applications. Journal of controlled release : official journal of the Controlled Release Society. 2008; 130: 202-15.

43. Zander J, Maier B, Zoller M, Dobbeler G, Frey L, Teupser D, et al. Effects of biobanking conditions on six antibiotic substances in human serum assessed by a novel evaluation protocol. Clin Chem Lab Med. 2016; 54: 265-74.

44. Esteban MJ, Canton E, Rius F. Influence of temperature on degradation kinetics of ceftriaxone in diluted and undiluted human serum. Antimicrob Agents Chemother. 1990; 34: 1268-70.

45. McLaren AC, McLaren SG, Nelson CL, Wassell DL, Olsen KM. The effect of sampling method on the elution of tobramycin from calcium sulfate. Clinical orthopaedics and related research. 2002: 54-7.

46. Mouton JW, Theuretzbacher U, Craig WA, Tulkens PM, Derendorf H, Cars O. Tissue concentrations: do we ever learn? The Journal of antimicrobial chemotherapy. 2008; 61: 235-7.

47. Schmutz HR, Detampel P, Buhler T, Buttler A, Gygax B, Huwyler J. in vitro assessment of the formation of ceftriaxone-calcium precipitates in human plasma. J Pharm Sci. 2011; 100: 2300-10.

48. Chutipongtanate S, Thongboonkerd V. Ceftriaxone crystallization and its potential role in kidney stone formation. Biochem Biophys Res Commun. 2011; 406: 396-402.

49. Shiffman ML, Keith FB, Moore EW. Pathogenesis of ceftriaxone-associated biliary sludge. in vitro studies of calcium-ceftriaxone binding and solubility. Gastroenterology 1990; 99: 1772-8.

50. Craig WA. Interrelationship between pharmacokinetics and pharmacodynamics in determining dosage regimens for broad-spectrum cephalosporins. Diagn Microbiol Infect Dis. 1995; 22: 89-96.

51. Zeitlinger M, Sauermann R, Fille M, Hausdorfer J, Leitner I, Muller M. Plasma protein binding of fluoroquinolones affects antimicrobial activity. The Journal of antimicrobial chemotherapy. 2008; 61: 561-7.

52. Adaway JE, Keevil BG. Therapeutic drug monitoring and LC-MS/MS. J Chromatogr B Analyt Technol Biomed Life Sci. 2012; 883-884: 33-49.

53. Bonev B, Hooper J, Parisot J. Principles of assessing bacterial susceptibility to antibiotics using the agar diffusion method. The Journal of antimicrobial chemotherapy. 2008; 61: 1295-301.

54. Rossaint R, Bouillon B, Cerny V, Coats TJ, Duranteau J, Fernandez-Mondejar E, et al. Management of bleeding following major trauma: an updated European guideline. Critical care. 2010; 14: R52. 
55. Srinivas NR. Dodging matrix effects in liquid chromatography tandem mass spectrometric assays--compilation of key learnings and perspectives. Biomed Chromatogr. 2009; 23: 451-4.

56. Keevil BG, Lockhart SJ, Cooper DP. Determination of tobramycin in serum using liquid chromatography-tandem mass spectrometry and comparison with a fluorescence polarisation assay. J Chromatogr B Analyt Technol Biomed Life Sci. 2003; 794: 329-35.

57. Holtom PD, Pavkovic SA, Bravos PD, Patzakis MJ, Shepherd LE, Frenkel B. Inhibitory effects of the quinolone antibiotics trovafloxacin, ciprofloxacin, and levofloxacin on osteoblastic cells in vitro. Journal of orthopaedic research : official publication of the Orthopaedic Research Society. 2000; 18: 721-7.

58. Antoci V, Jr., Adams CS, Hickok NJ, Shapiro IM, Parvizi J. Antibiotics for local delivery systems cause skeletal cell toxicity in vitro. Clinical orthopaedics and related research. 2007; 462: 200-6.

59. Rathbone CR, Cross JD, Brown KV, Murray CK, Wenke JC. Effect of various concentrations of antibiotics on osteogenic cell viability and activity. Journal of orthopaedic research : official publication of the Orthopaedic Research Society. 2011; 29: 1070-4.

60. Naal FD, Salzmann GM, von Knoch F, Tuebel J, Diehl P, Gradinger R, et al. The effects of clindamycin on human osteoblasts in vitro. Archives of orthopaedic and trauma surgery. 2008; 128: 317-23. 